Article

\title{
Identification of Differentially Expressed Genes between "Honeycrisp" and "Golden Delicious" Apple Fruit Tissues Reveal Candidates for Crop Improvement
}

\author{
Scott Schaeffer ${ }^{1,2,+}$, Christopher Hendrickson ${ }^{2, \ddagger}$, Rachel Fox ${ }^{2}$ and Amit Dhingra 1,2,* \\ 1 Molecular Plant Science Program, Washington State University, Pullman, WA 99164, USA; \\ scott.schaeffer@bcm.edu \\ 2 Department of Horticulture, Washington State University, Pullman, WA 99164, USA; \\ chendrickson@nu.edu (C.H.); rldaniel4@gmail.com (R.F.) \\ * Correspondence: adhingra@wsu.edu; Tel.: +1-509-335-3625 \\ + Current address: Department of Agriculture-Agriculture Research Service, \\ Children's Nutrition Research Center, Baylor College of Medicine, Houston, TX 77030, USA. \\ $\ddagger$ Current address: Department of Mathematics and Natural Sciences, National University, La Jolla, \\ CA 92037, USA.
}

Academic Editors: Lijun Wang and Douglas D. Archbold

Received: 1 February 2016; Accepted: 6 August 2016; Published: 17 August 2016

\begin{abstract}
Cultivars of the same species exhibit a large degree of variation in fruit quality traits, which can be directly influenced by differences in gene expression due to allelic variations and interactions with the environment. For Malus $\times$ domestica Borkh. (apple), fruit quality traits, including color, texture, aroma, flavor profile, and shelf life, are of utmost economic importance. In order to identify genes potentially influencing these traits, a direct comparative transcriptome profiling approach, based on the differential display technique, was performed using "Golden Delicious" and "Honeycrisp" apple endocarp and peel tissues. A total of 45 differentially expressed sequence tags were identified between the two apple varieties. Reanalysis of a previously published fruit developmental microarray expression experiment revealed that only one of the 45 sequence tags was represented on the array. Differential expression of 31 sequence tags from the peel tissue was validated using quantitative reverse transcription PCR, confirming the robustness of the differential display approach to quickly identify differentially expressed sequence tags. Among these were genes annotated to be involved in ripening, phytohormone signaling, transcription factors, and fruit texture. This work demonstrates yet again the utility of the differential display technique to rapidly identify genes related to desirable traits.
\end{abstract}

Keywords: differential display; differential expression; fruit quality; Malus $\times$ domestica Borkh

\section{Introduction}

Fruit quality traits vary across different cultivars of the same species during development, storage, and/or transit to market. In pome fruit, these traits can be broadly classified as peel or endocarp-specific. Peel-specific traits include fruit color, photo-protective capacity, scalding susceptibility, lenticel injury, aroma, etc. Endocarp-specific traits include propensity for rotting and mold development, hypoxic injury, flesh-sweetness, acidity, and textural qualities. Adding further complexity, these traits are under polygenic control and can vary by season, soil conditions and elevation [1-3].

Development of new tree fruit cultivars remains a laborious and time-consuming process. Juvenility periods and a perennial fruit-bearing nature can require at least five years to study the 
effect of genes in progeny of a controlled cross. Additionally, desirable traits in tree fruits may be highly dependent on genetic and environmental interactions during the course of fruit development and maturation, which can complicate attempts at performing uniform phenotyping. As a result, identification of causal genetic mechanisms underlying traits of interest in tree fruits can be difficult. Additionally, genomic structure of such species can compound challenges. Theorized duplication events have yielded allotetraploid genome structures in several perennial tree fruits including apple and pear $[4,5]$. This can result in numerous functionally redundant or interrelated families of genes involved in controlling traits of interest in tree fruit. To facilitate crop improvement in such systems, desirable genes must first be functionally validated prior to them being selectively introgressed into future genetic selections.

Variation in fruit quality traits can be influenced by numerous genetic factors including, but not limited to, allelic variation, gene copy number, and transcript variants. Differential gene and allelic expression may also result in aberrant folding or post-translational modification of proteins within the members of the same species [6,7]. While the causes of the phenotypic differences can vary, approaches used to identify causal genetic elements have been largely uniform. To identify the genetic elements, researchers rely upon segregation probabilities of DNA polymorphisms in populations segregating for the desired traits with the use of molecular markers such as RFLPs (Restriction Fragment Length Polymorphism), SNPs (Single Nucleotide Polymorphism), and microsatellites [8,9]. Environmental cues, polygenic control of quantitative traits, and allelic expression differences can complicate the use of molecular markers in identification of underlying genetic elements in an apple. For these reasons, the exclusive use of marker-based approaches to identify underpinnings of desirable fruit phenotypes can be challenging. While not feasible for all traits, a gene-linked mechanistic understanding of desirable fruit traits wherever possible may provide a reliable and reproducible resource for crop improvement. The Malus research community has developed and deployed molecular markers from densely populated maps of all Malus $\times$ domestica Borkh. linkage groups. Fruit firmness in apple was reported to be linked with the allelic composition of aminocyclopropane-1-carboxylic acid synthase (ACS) and oxidase (ACO) genes that regulate ethylene biosynthesis in climacteric fruit [10,11]. Zhu and Barrett [12] applied this information to nearly 100 apple lines and concluded that those homozygous for ACS1-2 and ACO1-1 had firmer fruit both at harvest and after typical commercial postharvest storage, compared to other genotypes. Additional correlations have been made between a Malus SSR marker and resistance to Erwinia amylovra (fireblight) [13]. These signatures of economically-important traits can be used to develop improved germplasm for the commercial market.

Recently, a wealth of additional tree fruit genomic resources have been established, with the release of the genomes from the "Golden Delicious" apple, sweet orange, peach, and Chinese white pear, as well as the recently announced European pear genomes [4,5,14-16]. These resources are enabling the understanding of genetic causes underlying numerous traits in tree fruit such as resistance to pathogens, fruit sweetness, acidity, texture, peel and flesh coloring, and responses to numerous postharvest storage regimes.

One of the less used approaches to identify the genetic basis for phenotypic differences between two varieties is to perform a direct comparison of gene expression in a spatio-temporal context. One such small scale approach for comparative transcriptome profiling is the differential display reverse transcriptase polymerase chain reaction, or DD [17]. The DD technique can amplify all poly-adenylated transcripts allowing for direct capture of actively expressed genes and alleles in a comparative context. The resulting gene fragment amplicons can be isolated based upon either their presence or absence, or based on differential intensity on a polyacrylamide gel that is indicative of differential expression. Identified fragments are cloned, sequenced, and annotated to infer their biological function. This approach has been utilized in many plant systems to identify putative targets involved in many traits or processes including reproductive roles in semigametic Pima cotton (Gossypium barbadense), heat responsive genes in peach (Prunus persica), hormone and abiotic stress changes in the gene expression of tea (Camelina sinensis), and ripening-related genes in banana 
(Musa acuminata AAA) [18-21]. In each of these instances, DD aided in direct identification of genes potentially involved in the regulation of the phenotypes being studied.

Despite the labor and time-related challenges associated with performing a DD approach, the technique retains some significant advantages over newer next-generation sequencing based approaches. Differential display offers an inexpensive means of identifying candidate genes without the complexities associated with RNA sequencing (RNA-seq) based approaches. The presence or absence of a PCR amplicon in a DD polyacrylamide gel provides a positive result that does not require a reference genome and advanced computational resources associated with comparative RNA-seq workflows. Further, DD can aid in the identification of potentially important regulatory genes between samples which can be overlooked using microarray-based comparisons. The complex data capture, assembly and statistical analysis associated with microarray and next-generation sequencing approaches can result in missing or misidentifying the genes that control traits of interest. Caution is prescribed in carefully examining novel transcripts identified via RNA-seq before proceeding to biological experiments.

Available apple germplasm abounds in diversity of fruit quality traits. The apple cultivar "Honeycrisp" (HC) falls into a category of "JFC high quality" indicating a juicy and crispy-textured flesh, while "Golden Delicious" (GD) is classified as "American/European dessert". HC is typified by a color profile which is $60 \%$ orange/red on top of a yellow base color, large size, as well as having an exceptional texture and juiciness [22]. GD, on the other hand, has a completely yellow color and texture that is juicy, firm and crisp. HC apples maintain a crisp texture up to 6 months after harvest even without controlled atmosphere conditions due to maintenance of cell wall integrity and cellular turgor potential [23]. These two apple varieties are popular among consumers, but exhibit highly diverse fruit quality characteristics through ontogeny and ripening. In this study, we investigated the differences in the expression of peel and flesh specific genes of GD and HC apples at two different developmental stages. Results obtained from this work identified several important genes related to fruit quality and demonstrate the utility of an inexpensive method for direct identification of candidate genes that could be utilized for development of improved apple varieties.

\section{Materials and Methods}

\subsection{Sampling Strategy}

HC and GD apples were harvested during two growing seasons at 125 or 129 and 150 or 160 days after anthesis (DAA), respectively. The time points sampled in this study were chosen due to their correlation with early and late stages of the ripening process in an apple, which typically begins around 95 days after anthesis (DAA) and concludes at around 150 DAA [1]. Additionally, these samples represent apple fruit at pre-climacteric and climacteric stages of maturity [2]. Tissue processing was performed directly in the orchard where the peel and endocarp samples were obtained from nine apples derived from three trees of the same variety. Samples were immediately frozen in liquid nitrogen. Tissues were stored at $-80^{\circ} \mathrm{C}$ and subsequently ground for three cycles of four minutes at top speed using a SPEX SamplePrep 6870 Freezer mill (Metuchen, NJ, USA). Total RNA was extracted from ground tissue using Plant RNeasy Minikit according to the manufacturer's instructions (Qiagen, Valencia, CA, USA). RNA was quantified using a Nanodrop 8000 spectrophotometer (Wilmington, DE, USA) and its integrity was confirmed with a denaturing formaldehyde MOPS gel.

\subsection{Differential Display}

Differential displays were performed on HC endocarp, HC peel, GC endocarp and GC peel samples. Removal of DNA contamination from RNA was performed with the MessageClean Kit (GenHunter, Nashville, TN, USA) according to manufacturer's instructions. Synthesis of cDNA and PCR amplification was performed using the GenHunter RNAimage Kit (GenHunter) according to the manufacturer's instructions. Primers 193 to 200 (GenHunter) in combination with the anchoring 
poly-T, $\mathrm{H}-\mathrm{T}_{11} \mathrm{~A}$, were used for PCR amplification. ${ }^{33} \mathrm{P}$-labeled cDNA fragments were separated on a $6 \%$ acrylamide denaturing gel. After imaging through radiography, bands were excised if differential expression was observed between HC and GD tissues.

\subsection{Cloning and Sequencing}

Excised bands were boiled for 10 min with $40 \mu \mathrm{L}$ of deionized water in a $1.5 \mathrm{~mL}$ microcentrifuge tube. Re-amplification was performed using $1 \mu \mathrm{L}$ of the recovered DNA as a template and the differential display primers used for their initial amplification. PCR amplicons were cleaned using the QIAquick PCR Purification Kit (Qiagen) and subsequently ligated into the pGEM-T Easy vector (Promega, Madison, WI, USA) according to the manufacturer's protocol. Ligated fragments were then transformed into Escherichia coli XL1-Blue cells via the heat-shock method. Colony PCR was used to confirm the presence of an insert using M13 forward and reverse primers. Plasmids were sequenced using Sanger sequencing with M13 forward primers at Eurofins MWG Operon. Gene IDs as predicted from the apple genome of the sequenced fragments are provided in Table S1.

\subsection{Annotation of Differential Display Fragments}

BLASTN was performed for all sequenced differential display fragments against the predicted gene sequences from the apple genome release 1.0. As differential display sequences were derived from the 3' end of transcripts and could contain untranslated regions, a second BLASTN was performed against the NCBI EST database for Rosaceae sequences (NCBI taxid: 3745). Matching EST sequences were subsequently mapped to full length apple gene sequences with BLASTN which were extracted for subsequent analysis. All full length apple gene sequences were then analyzed by Blast2GO against the NCBI nr database to determine putative functions [3,4]. Localization of each protein sequence was performed with TargetP using plant networks with default parameters [5]. A final BLASTX step was performed against the NCBI protein collections from Arabidopsis thaliana and Solanum lycopersicum to identify homologs in these systems, with the top result from each species with an E-value of at least $1 \mathrm{E}^{-20}$ listed. The description, if more detailed than the original Blast2GO hit, was also retained.

\subsection{Quantitative Real Time PCR Validation of Differential Expression}

RNA from HC and GD peel tissue collected over two growing seasons was extracted as described above. Validation of differential expression using qPCR was performed with peel tissues. Samples were DNAse-treated using the DNA-free kit (Ambion) according to the manufacturer's instructions. RNA was quantified using Nanodrop 8000 and its integrity was tested on a $1.0 \%$ agarose gel. First-strand cDNA was synthesized using the SuperScript VILO cDNA Synthesis Kit (Life Technologies, Carlsbad, CA, USA) following manufacturer's instructions. Concentration was diluted to $50 \mathrm{ng} / \mu \mathrm{L}$ with deionized water. Validation of differential expression was performed for 31 apple genes: MDP0000200646, MDP0000037251, MDP0000875654, MDP0000296716, MDP0000128468, MDP0000712586, MDP0000618650, MDP0000920333, MDP0000152947, MDP0000883284, MDP0000253074, MDP0000213808, MDP0000547450, MDP0000310811, MDP0000232309, MDP0000161275, MDP0000176723, MDP0000304285, MDP0000172863, MDP0000180389, MDP0000523205, MDP0000138340, MDP0000166116, MDP0000220601, MDP0000237908, MDP0000273484, MDP0000286959, MDP0000292888, MDP0000320533, MDP00005772242, and MDP0000697474. Primers for PCR were designed with the assistance of custom scripts based on predicted gene sequences from the apple genome project. These primers were then used in quantitative reverse-transcription PCR validation of DD results using iTaq Universal SYBR Green Supermix (Bio-Rad Laboratories, Hercules, CA, USA) on a Stratagene MX3005P light cycler (Agilent, Santa Clara, CA, USA). All reactions were performed using $50 \mathrm{ng}$ cDNA and carried out in triplicate along with a $\beta$-tubulin internal control. $\beta$-Tubulin was selected as it has been previously used as an internal control in several prior apple gene expression studies [6-8]. Efficiencies and Cq values were determined using the tool LinRegPCR [9]. Any reactions with an efficiency less than 1.8 or more than 2.2 were discarded from further analysis. Cq values were compared between the three replicates and, 
in the case that the standard deviation exceeded one, one of the three outliers was omitted from the analysis. The Pfaffl correction was then applied to $\mathrm{Cq}$ values of remaining reactions. Fold change was determined by comparing the highest $\mathrm{Cq}$ value to a $\beta$-tubulin internal control [10]. To validate the identity of the genes, 10 random qPCR products were sequenced. These included MDP0000312808, MDP0000232309, MDP00000320533, MDP0000547450, MDP0000697474, MDP0000166116, MDP0000253074, MDP0000166116, MDP0000253074, MDP0000255887, MDP0000200646, and MDP0000920333. Products were sequenced using the forward and reverse primers that were originally used to amplify them. Sequences were assembled using Lasergene 11 SeqMan Pro (DNASTAR, Madison, WI, USA) and compared to the full length apple gene using a pairwise BLAST.

\subsection{Comparison of Expression with Previous Studies}

Previously generated "Royal Gala" apple microarray expression data published in 2008 [1] was reanalyzed to identify data from genes recognized in the differential display experiment. All ESTs represented in the experiment were compared to the predicted apple gene sequences generated from the apple genome with BLASTN. The top hits with an e-value lower than $1 \mathrm{E}^{-10}$ were then assigned the apple gene identifiers. These identifiers were then compared with the genes represented in the differential display experiment.

\section{Results and Discussion}

\subsection{Differential Display}

Apple ripening is characterized by the conversion of starches to simple sugars, decrease of total chlorophyll and photosynthetic activity, increase in total carotenoids, flesh softening and cell wall modification as well as accumulation of volatiles and flavor compounds [11-13]. Any differences in these properties would likely be mirrored in differences in gene expression. Differential display analysis of the peel tissues from two developmental stages of GD and HC tissues in apple, generated 115 bands corresponding to potentially differentially expressed genes. Of these, 105 fragments could be isolated which were then cloned into a plasmid and sequenced. The remaining ten could not be re-amplified after extraction from the PAGE (Polyacrylamide Gel Electrophoresis). BLASTN was performed for each sequence against the predicted gene set from the apple genome as well as the NCBI EST sequences available for Rosaceae (NCBI taxid: 3745). The apple genome and Rosaceae EST sequences similar to the 105 query sequences were extracted and processed through BLAST2GO workflow and the output is available in Table S2. For each matching EST sequence, a final BLAST was performed against the apple predicted gene set. This analysis resulted in the identification of 42 sequences from the differential display experiment, which had significant identity with a full-length apple gene. Three of these sequences, however, had different apple gene matches between the two BLAST methods. In order to account for this discrepancy, all predicted matches were included resulting in 45 sequences which were chosen for further characterization and validation.

Blast2GO analysis was performed on matching full length apple sequences to predict potential functions associated with the sequences, while TargetP was used to predict the cellular localization of each protein (Table 1) [3-5,14]. Of these genes, seven are predicted to be secreted from the cell, while fewer are predicted to be localized to the mitochondria and the chloroplast. The most represented molecular functions in this group include transporter activity (GO:0005215, four sequences), sequence-specific DNA binding transcription factor activity (GO:0003700, three sequences), and protein binding (GO:0005515, three sequences). Relatively more information was available for the biological processes in which these proteins may participate, which include response to stress (GO:0006950, 11 sequences), response to biotic stimulus (GO:0009607, nine sequences), transport (GO:0006810, eight sequences), and catabolic process (GO:0009056, seven sequences). Molecular function and biological process gene ontologies for these genes are summarized in Figure 1. A final BLASTX was performed against the NCBI protein collections from Arabidopsis thaliana and Solanum lycopersicum to identify the homologs in these systems (Table 2). 
Table 1. Identification of fragments isolated from an initial differential display procedure. Differential expression was assessed using "Honeycrisp" and "Golden Delicious" apple fruit peel and endocarp (HCP, HCE, GDP, and GCE, respectively) collected at 129 and 160 days after anthesis (DAA) in the 2007 growing season. Bands from differential display gels were excised, cloned and sequenced. Qualitative abundance was determined for each tissue and noted as either absent $(-)$ present $(+)$ or overexpressed (++). Sequences were matched to predicted apple genes via BLAST and analyzed for predicted localization using TargetP (M—mitochondria, C—chloroplast, S—secreted,_-no localization).

\begin{tabular}{|c|c|c|c|c|c|c|c|c|c|c|}
\hline \multirow{2}{*}{ Gene Identifier } & \multirow{2}{*}{ Blast2GO Annotation } & \multicolumn{4}{|c|}{129 DAA-2007 Season } & \multicolumn{4}{|c|}{160 DAA-2007 Season } & \multirow{2}{*}{$\begin{array}{c}\text { Cellular } \\
\text { Localization }\end{array}$} \\
\hline & & GDE & GDP & HCE & HCP & GDE & GDP & HCE & HCP & \\
\hline MDP0000037251 & cinnamyl alcohol dehydrogenase-like protein & ++ & ++ & + & ++ & ++ & ++ & + & ++ & \\
\hline MDP0000128468 & abscisic acid stress ripening protein homolog & ++ & ++ & ++ & + & ++ & ++ & ++ & + & $\mathrm{M}$ \\
\hline MDP0000129664 & 3-ketoacyl-thiolase & - & - & + & + & - & - & + & + & _- \\
\hline MDP0000138340 & NAC domain ipr003441 & ++ & ++ & + & + & ++ & ++ & + & + & - \\
\hline MDP0000152947 & wound-induced protein & + & ++ & + & + & + & + & + & + & _- \\
\hline MDP0000161275 & mitochondrial substrate carrier family protein & + & + & + & ++ & + & + & + & ++ & _- \\
\hline MDP0000166116 & acyl:CoA ligase acetate-coa synthetase-like protein & + & + & + & + & ++ & ++ & +++ & +++ & - \\
\hline MDP0000172863 & protein & - & - & - & - & - & + & - & - & $\overline{\mathrm{S}}$ \\
\hline MDP0000176723 & acyl:CoA ligase acetate-coa synthetase-like protein & ++ & ++ & + & + & ++ & ++ & + & + & 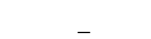 \\
\hline MDP0000180389 & disease resistance protein at3g14460-like & ++ & ++ & + & + & ++ & ++ & + & + & $\bar{S}$ \\
\hline MDP0000200646 & NAC domain ipr003441 & + & + & + & + & + & + & ++ & ++ & _- \\
\hline MDP0000213808 & probable ubiquitin conjugation factor e4-like & ++ & ++ & + & + & ++ & ++ & + & + & _- \\
\hline MDP0000220601 & zinc finger $\mathrm{CCCH}$ domain-containing protein 53-like & + & + & + & + & + & + & + & ++ & - \\
\hline MDP0000232309 & transmembrane $\mathrm{BAX}$ inhibitor motif-containing protein 4 & + & + & + & + & ++ & ++ & + & + & _- \\
\hline MDP0000233229 & Unknown protein & + & + & + & + & + & + & + & ++ & _- \\
\hline MDP0000234325 & WWE protein-protein interaction domain family protein & - & - & + & - & - & - & + & - & - \\
\hline MDP0000237908 & metallothionein-like protein & ++ & ++ & + & + & ++ & ++ & + & + & _ \\
\hline MDP0000253074 & abscisic acid stress ripening protein homolog & - & - & + & + & - & - & + & + & - \\
\hline MDP0000255887 & TIR-NBS-LRR resistance protein & - & + & - & - & - & + & - & - & - \\
\hline MDP0000273484 & SKP1-like protein & - & + & - & - & - & + & - & - & - \\
\hline MDP0000281279 & Unknown protein & ++ & + & + & + & ++ & + & + & + & - \\
\hline MDP0000286959 & dentin sialophosphoprotein & + & + & - & - & + & + & - & - & - \\
\hline MDP0000292888 & GYF domain-containing protein & - & - & - & + & - & - & - & + & $\overline{\mathrm{C}}$ \\
\hline MDP0000296716 & ethylene-responsive transcription factor RAP2-7-like & ++ & + & ++ & ++ & ++ & + & ++ & ++ & _ \\
\hline MDP0000304285 & xanthine uracil permease family expressed & - & - & - & + & - & - & - & + & - \\
\hline
\end{tabular}


Table 1. Cont

\begin{tabular}{|c|c|c|c|c|c|c|c|c|c|c|}
\hline \multirow{2}{*}{ Gene Identifier } & \multirow{2}{*}{ Blast2GO Annotation } & \multicolumn{4}{|c|}{129 DAA-2007 Season } & \multicolumn{4}{|c|}{160 DAA-2007 Season } & \multirow{2}{*}{$\begin{array}{c}\text { Cellular } \\
\text { Localization }\end{array}$} \\
\hline & & GDE & GDP & HCE & HCP & GDE & GDP & HCE & HCP & \\
\hline MDP0000310811 & cysteine protease inhibitor & - & - & - & - & - & - & - & + & $\mathrm{M}$ \\
\hline MDP0000316244 & probable ADP-ribosylation factor GTPase-activating protein AGD15-like & + & + & ++ & ++ & ++ & ++ & + & + & _ \\
\hline MDP0000320533 & proteasome assembly chaperone & - & - & - & + & - & - & - & + & - \\
\hline MDP0000378585 & at4g03420 f9h3_4 & + & + & ++ & + & + & + & ++ & + & \\
\hline MDP0000443265 & Unknown protein & - & - & - & - & - & - & + & - & $\overline{\mathrm{C}}$ \\
\hline MDP0000523205 & Unknown protein & - & - & - & - & - & + & - & - & _- \\
\hline MDP0000547450 & UNC93-like protein & + & ++ & + & + & + & ++ & + & + & - \\
\hline MDP0000572242 & probable xyloglucan glycosyltransferase 12 -like & + & ++ & + & + & + & ++ & + & + & - \\
\hline MDP0000580900 & porin voltage-dependent anion-selective channel protein & + & - & - & - & + & - & - & - & - \\
\hline MDP0000584042 & protein & + & + & - & - & + & + & - & - & $\bar{S}$ \\
\hline MDP0000606453 & probable nitrite transporter at $1 \mathrm{~g} 68570$-like & - & - & - & - & - & - & + & - & - \\
\hline MDP0000618650 & NAC domain ipr003441 & - & - & - & + & - & - & - & + & - \\
\hline MDP0000689999 & protein disulfide isomerase & ++ & + & + & + & ++ & + & + & + & $\overline{\mathrm{S}}$ \\
\hline MDP0000697474 & reverse transcriptase & - & + & - & - & - & + & - & - & \\
\hline MDP0000712586 & protein SCAI-like & + & + & ++ & ++ & ++ & ++ & + & + & $\overline{\mathrm{S}}$ \\
\hline MDP0000875654 & hydroquinone glucosyltransferase & ++ & ++ & + & + & + & + & + & + & _- \\
\hline MDP0000876817 & Unknown protein & - & - & - & - & - & - & + & - & - \\
\hline MDP0000883284 & PREDICTED: uncharacterized protein LOC100248602 [Vitis vinifera] & + & + & - & - & + & + & - & - & $\overline{\mathrm{S}}$ \\
\hline MDP0000901731 & Unknown protein & - & - & - & + & - & - & - & + & $\mathrm{s}$ \\
\hline MDP0000920333 & putative protein phosphatase $2 \mathrm{C} 10$ & - & - & - & + & - & - & - & + & _ \\
\hline
\end{tabular}


Table 2. Linking differentially expressed genes to changes in fruit traits. Functional annotation for differentially expressed genes was performed using Blast2GO, and additional BLASTX was performed against the proteomes of Arabidopsis thaliana and Solanum lycopersicum. Many of the differentially expressed genes influence important traits within the fruits.

\begin{tabular}{|c|c|c|c|c|c|c|}
\hline Gene ID & Location & Gene & At Homolog & S1 Homolog & $\begin{array}{c}\text { Potential Trait/Fruit } \\
\text { Characteristic Influenced }\end{array}$ & Reference(s) \\
\hline \multicolumn{7}{|c|}{ Transcriptional Regulation } \\
\hline MDP0000138340 & chr1:12,793,865..12,795,081 & NAC transcription factor-like 9 & AT4G35580 & XP_004239709.1 & Stress response & [15] \\
\hline MDP0000200646 & chr1:12,796,087..12,797,315 & NAC transcription factor-like 9 & AT4G35580 & XP_004239709.1 & Stress response & [15] \\
\hline MDP0000618650 & $\operatorname{chr15:14,098,214..14,102,470}$ & NAC domain containing protein 75 & AT4G29230 & XP_004239596.1 & Stress response/general ripening regulation & [15] \\
\hline MDP0000296716 & chr3:9,357,237..9,360,502 & AP2 transcription factor SIAP2d & AT2G28550 & NP_001234647.1 & Ethylene-regulated response & {$[16,17]$} \\
\hline \multicolumn{7}{|c|}{ Secondary Biosynthetic Processes } \\
\hline MDP0000037251 & chr1:6,887,281..6,890,898 & Putative cinnamyl alcohol dehydrogenase 9 & AT4G39330 & XP_004250169.1 & Fruit firmness & [18] \\
\hline MDP0000572242 & chr15:1,231,253..1,235,097 & Probable xyloglucan glycosyltransferase 12-like & AT4G07960 & XP_004238064.1 & Fruit firmness & [19] \\
\hline MDP0000875654 & chr7:16,340,110..16,341,528 & Hydroquinone glucosyltransferase & AT4G01070 & XP_004231207.1 & Volatile/polyphenol glycosylation & {$[20,21]$} \\
\hline MDP0000523205 & chr7:5,077,703..5,080,814 & Flavonoid biosynthesis oxidoreductase protein & AT4G10490 & NP_001233840.1 & Flavanoid synthesis & [22] \\
\hline \multicolumn{7}{|c|}{ Signaling } \\
\hline MDP0000128468 & chr16:4,935,225.4, 935,891 & Abscisic acid stress ripening (ASR1) protein & No hit & NM_001247208.2 & $\begin{array}{l}\text { Abiotic stress response, developmental } \\
\text { response to sugar levels }\end{array}$ & {$[23,24]$} \\
\hline MDP0000176723 & 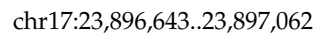 & Acyl:CoA ligase acetate-CoA synthase-like protein & AT5G16370 & XP_004231630.1 & Abiotic stress response/ripening & [25-27] \\
\hline MDP0000920333 & $\operatorname{chr10:29,789,438..29,789,994}$ & Putative protein phosphatase $2 \mathrm{C}-10$ & AT1G34750 & XP_004237914.1 & Ripening/abiotic stress response & {$[28,29]$} \\
\hline MDP0000213808 & chr17:4,103,927..4,109,528 & putative ubiquitin conjugation factor E4 & AT5G15400 & XP_004232186.1 & Ethylene production, development, ripening & [30] \\
\hline \multicolumn{7}{|c|}{ Cell/Organ Development } \\
\hline MDP0000232309 & 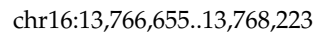 & BAX inhibitor-1 like protein & No hit & No hit & Cell elongation/fruit size & {$[31,32]$} \\
\hline MDP0000547450 & $\operatorname{chr} 7: 26,032,235 . .26,033,783$ & UNC93-like protein 1-like & AT1G18000 & XP_004235041.1 & Apogamy & [33] \\
\hline \multicolumn{7}{|c|}{ Stress Response } \\
\hline MDP0000712586 & chr14:27,117,925..27,120,988 & SCAI-like protein & No Hit & No Hit & Redox homeostasis/respiration & [34] \\
\hline MDP0000161275 & $\operatorname{chr12:24,524,346..24,526,137}$ & Mitochondrial succinate-fumarate transporter 1-like & AT5G01340 & XP_004249636.1 & Hypoxia-induced fermentation/respiration & {$[35,36]$} \\
\hline MDP0000310811 & chr7:3,004,269..3,005,959 & Cysteine proteinase inhibitor 6 & AT3G12490 & XP_004228480.1 & Abiotic stress tolerance & {$[37,38]$} \\
\hline
\end{tabular}


Sequence distribution: biological_process(Filtered by \#Seqs: cutoff $=1.0$ )

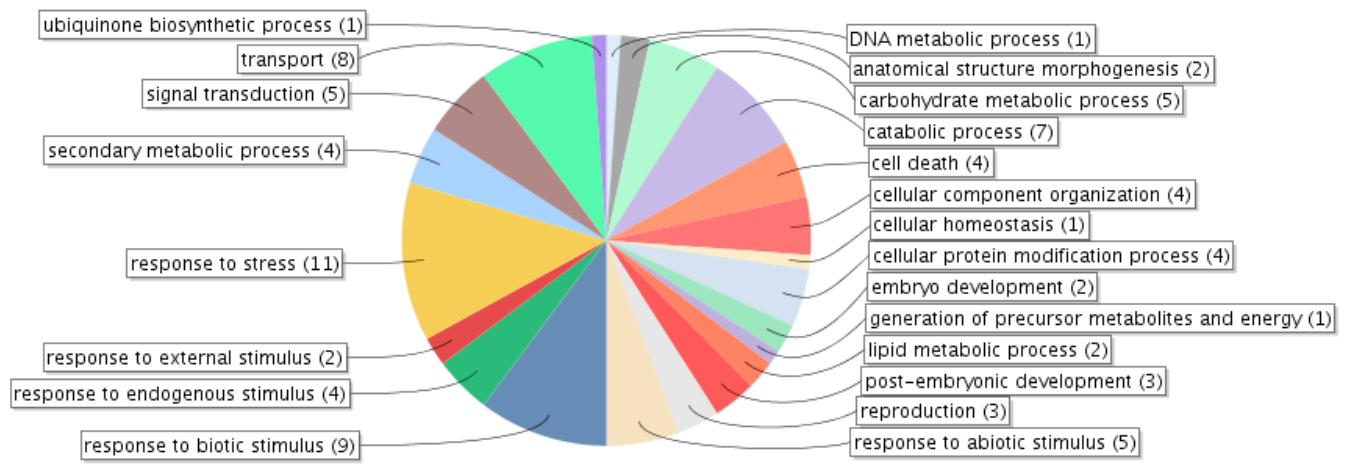

Sequence distribution: molecular_function(Filtered by \#Seqs: cutoff =1.0)



Figure 1. GO-terms associated with excised and sequenced bands from the differential display procedure. Functional analysis of sequences was performed using the Blast2GO annotation suite from full length apple genome (v. 1.0) sequences to which differential display band sequences were matched via BLAST.

\subsection{Quantitative Reverse Transcription PCR Validation of Differential Expression}

Differential expression observed in the differential display experiment was validated using quantitative reverse-transcription PCR (qPCR). This was performed for all samples when at least one peel tissue exhibited differential gene expression. A second biological replicate from the second growing season was also used to test for seasonal variation. Primer sets were designed based upon predicted gene sequence custom script which selects 26-mer primers, and screens the entire reference genome to ensure the specificity of the oligonucleotide. Primer sequences are provided in Table S3. Expression analysis was performed in triplicate for each gene along with a $\beta$-tubulin control which exhibited a global standard deviation of $<1.0$ across all replicates making it an ideal reference gene. Validation of qPCR specificity was performed by selecting ten differentially expressed genes and sequencing the qPCR products from GD and HC separately. Nine qPCR products aligned with the predicted sequences that were used for the primer design, while product for one, MDP0000255887, failed to yield a consensus sequence or match the original gene.

Analysis of the resulting amplification plots from qPCR revealed that the expression of MDP0000129664 (3-ketoacyl-thiolase) and MDP0000255887 (TIR-NBS-LRR resistance protein) were detected within the set threshold (Cq value lower than 35) in only some of the tested tissues. This could explain why sequences from the GPCR products of MDP0000255887 failed to yield appropriate sequence data. Additionally, no transcripts were detected for MDP0000233229 (protein) and MDP0000901731 (unknown) in any of the samples investigated. Further analysis of the expression for these genes was not pursued.

For the 31 genes whose expression was validated with qPCR, differential expression for each gene was observed, with at least one tested sample having a two-fold increase. Differential gene expression was separated into four categories: high differential expression (greater than five-fold change in 
expression) (Figure 2), moderate differential expression (three to five-fold change in expression) (Figure 3), low differential expression (zero to three-fold change in expression) (Figure S1), and seasonally-variable differential expression (Figure S2). The largest differences in gene expression were seen in MDP0000618650 (NAC domain containing protein), MDP0000875654 (hydroquinone glucosyltransferase), MDP0000037251 (cinnamyl alcohol dehydrogenase), MDP0000712586 (SCAI-like protein), MDP0000883284 (unknown), MDP0000200646 (NAC domain containing protein), and MDP0000920333 (protein phosphatase 2C). All of these genes were expressed at a higher level in GD compared to HC.
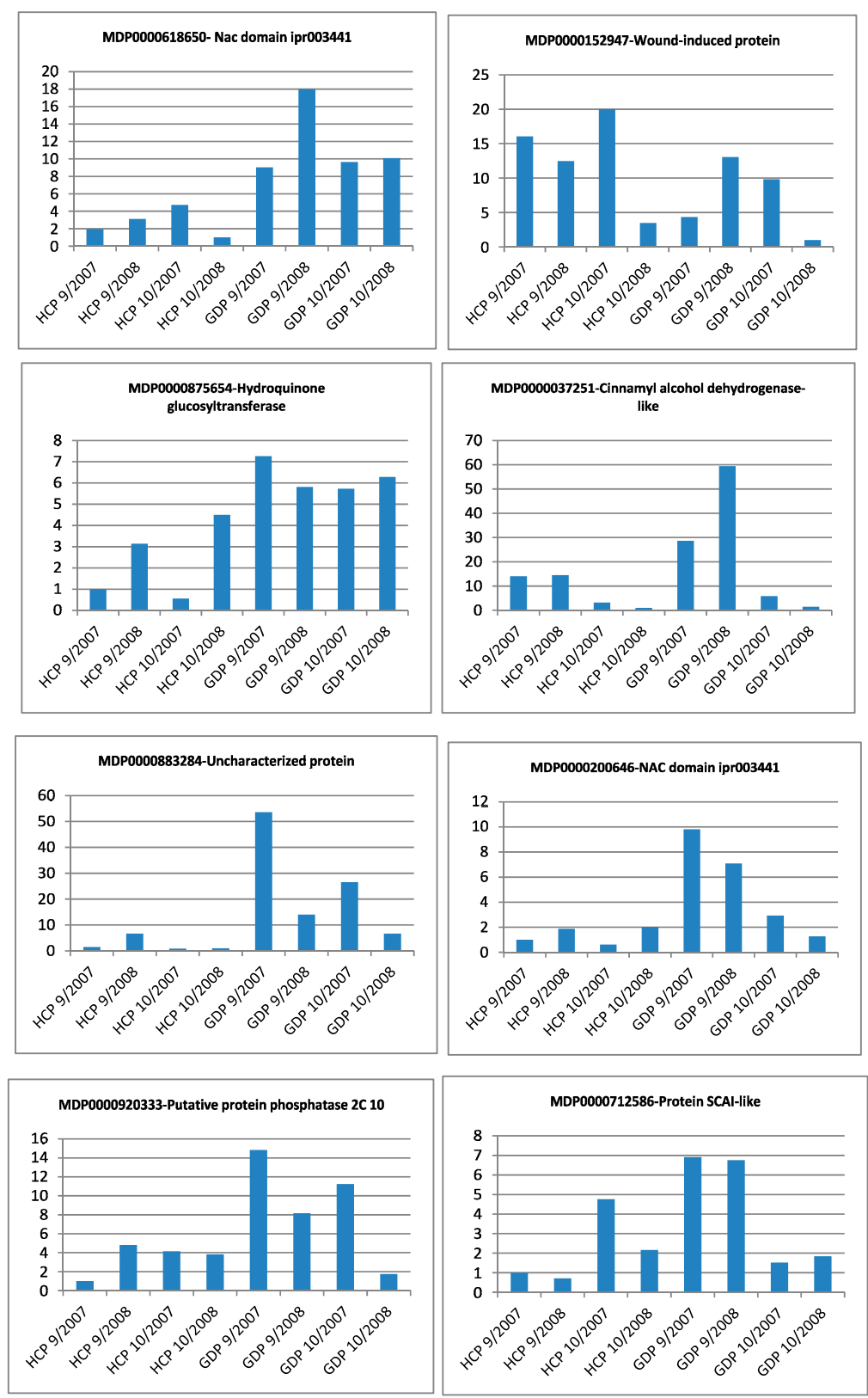

Figure 2. "High" differential expression of genes identified with the differential display procedure. Genes whose expression was greater than or equal to five-fold higher in expression between "Golden Delicious" and "Honeycrisp" peel tissue were classified as highly differentially expressed. Expression is separated by samples tested and plotted against fold expression relative to a $\beta$-tubulin internal control. 

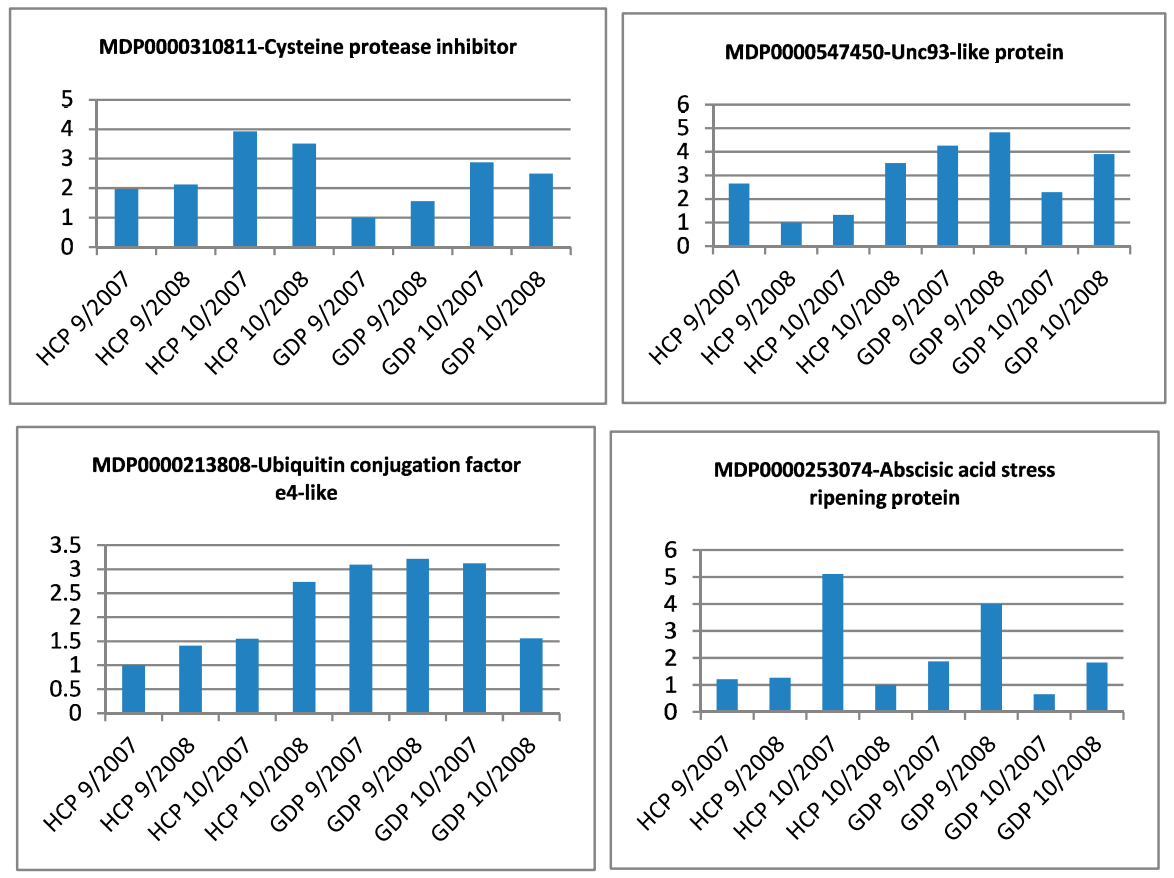

Figure 3. "Moderate" differential expression of genes identified with differential display. Samples with three to five-fold differences in expression relative to the control were classified as moderately differentially expressed. Relative expression relative to a $\beta$-tubulin internal control is plotted in each unique biological sample.

\subsection{Comparison of Expression with Previous Studies}

In order to determine if the expression of the genes identified in this study have been profiled in greater detail in apple fruit, data obtained previously from developing "Royal Gala" tissue was reanalyzed [1]. This study reported the expression of 13,000 unigenes across eight developmental stages. BLASTN was performed for all EST sequences used in the microarray study against the predicted genes from the apple genome project. This study identified 7265 unique genes which were represented in the microarray study. Presence and absence in the DD study was then determined for all sequences represented in the microarray study. Of the 45 genes identified through DD, only MDP0000292888 (GYF domain-containing protein) was represented in the microarray study. This gene was most highly expressed at zero days after anthesis (DAA) with one and a half-fold higher expression compared to the other seven time points, suggesting stable expression over time in "Royal Gala".

When comparing the DD approach with a microarray approach such as that described in the Janssen study referenced earlier, this study showed that the DD approach was quite useful in identifying differentially expressed genes. Only one of the 45 genes represented in the DD dataset was described in the Janssen study, demonstrating that a more thorough transcriptomic profiling of multiple apple genotypes may help in furthering our knowledge of differential gene expression in apple fruit. It appeared that the most significant differences in expression were primarily derived from GD tissues. This could be in fact due to a bias of using the GD predicted gene sequences for designing primer sequences. As genomic and transcriptomic resources for $\mathrm{HC}$ increase, additional information from fragments corresponding to the initially isolated differential display bands may come to light.

\subsection{Differentially Expressed Homologs in Heterologous Systems}

In this study we identified several genes with differential gene expression between HC and GD peel tissues. Differences in the expression of these genes may have important influences upon fruit characteristics throughout the course of development and ripening. Interestingly, some of the differentially expressed genes between GD and HC have been studied in heterologous systems, and 
are linked or related to major fruit quality traits and regulatory pathways [39,40]. These genes have been linked to various processes including biotic and abiotic stress tolerance, secondary metabolism, signaling, cell or organ development, and transcriptional regulation (Table 2). Such variability may have a significant influence on fruit characteristics and be involved in the phenotypic diversity between HC and GD apples.

Two differentially expressed genes identified in this study, cinnamyl alcohol dehydrogenase (CAD) (MDP0000037251) and xyloglucan transferase (or xyloglycan endotransglycosylase) (MDP0000572242), may have significant effects on cell wall modification. These modifications can have drastic effects upon the texture of fruits either by increasing firmness or promoting loosening of cell walls. Within strawberry fruit, CAD has been previously shown to be localized to lignified cells [41]. Differential expression studies performed with strawberry genotypes varying in fruit firmness found CAD and cinnamyl CoA reductase to have the highest difference in expression in a microarray representing 1701 strawberry ESTs [42]. CADs have also been associated with lignification and consequently increased firmness in loquat (Eriobotrya japonica) [18]. Xyloglycan endotransglycosylase (XETs), on the other hand, modify xyloglucans which are hemicelluloses that link together cellulose microfibrils. XETs have been found in multiple isoforms, aiding either in cell expansion during fruit growth or softening during fruit ripening [19]. These enzymes have been associated with fruit softening in kiwi (Actinidia deliciosa), tomato (Solanum lycopersicum), and other fruits [43,44]. At 129 DAA GD exhibited higher expression of both of these genes compared to HC. At 160 DAA there was a drastic reduction in expression. The expression of CAD decreased in ripening fruit, as it was approximately 12-fold higher at 129 DAA compared to 160 DAA in HC apples, and 28 to 60-fold higher at 129 DAA than at 160 DAA in GD. While the reduction in expression between the time points could correlate with fruit softening associated with ripening, the drastic differences in expression between cultivars could be related to differences in fruit texture. Further studies on the functional analysis of the XET allele in the two cultivars could be used to test this association.

Two differentially expressed genes in this study, MDP0000523205 (homologous to Solanum lycopersicum flavonoid biosynthesis oxidoreductase protein) and MDP0000875654 (hydroquinone glucosyl transferase), may be involved in important biosynthetic activities in apple fruit. In apples alone, there are over 300 compounds linked to the aroma profile, with few enzymes involved in their synthesis presently characterized [11]. Hydroquinone glucosyl transferase is a putative target for volatile and phenolic-compound glycosylation. Glucosyl transferases have been previously shown to be involved in the glycosylation of plant volatiles in tomato and have been shown to have relaxed substrate specificity [20]. A hydroquinone glycosyl transferase has also been linked to the synthesis of the phenolic compound arbutin, known to act as an antimicrobial agent and maintain potential anticarcinogenic effects [45]. Flavonoid biosynthesis oxidoreductase proteins (flavanone-3-hydroylases) are important components of the flavonoid biosynthetic pathway in apples which lead to the production of proanthocyanidins [22]. While further research on biochemical activity of these specific enzymes will need to be performed, differential expression of the genes encoding these proteins reveals targets for differences in volatile composition, phenolic compound composition, and flavonoid content between the two cultivars.

Additionally, two auxin-responsive genes were identified that differed between GD and HC tissues. MDP0000176723 (AAE18) is thought to play a role in response to auxin precursors (indole-3-butyric acid, IBA) in the peroxisome, and may modulate available IBA via amino acid conjugation for downstream processing into active IAA [46-49]. With recent focus on the roles of auxin in fruit ripening, this presents a powerful link between peroxisome metabolites and auxin accumulation as apple fruit undergo ripening. Future research may clarify the influence of this relationship in pre-climacteric and ripening apple fruit. Similarly, protein phosphatase 2C-10 (PP2C-10, MDP0000920333) transcripts were found in higher abundance in GD peel than in HC peel. PP2C-10 is reported to function as a serine/threonine phosphatase and is thought to be responsive to active auxin in the cell [28]. While current understanding of these proteins in fruit ripening remains unclear, PP2C 
proteins have been implicated in MAPK cascades, calcium-calmodulin signaling, and ABA-signaling in the cell which all play a role in numerous ripening-related pathways [29]. More studies of PP2C genotypic and expression variability are needed to determine implications of this gene's expression on fruit quality in pre and postharvest conditions.

Ethylene is a critical plant hormone in modulating many late-stage fruit maturation and ripening processes in apples. Climacteric fruit exhibit an autocatalytic phase of ethylene biosynthesis. This behavior allows ethylene to function both as a trigger and a result of fruit ripening. An ethylene-responsive transcription factor RAP2-7-like transcript (MDP0000296716) was found in higher abundance in GD peels then in HC peel tissues in this study. Containing an AP2/ERF-like domain, RAP2-7 homologs are highly induced during various abiotic stresses and during floral development $[16,17]$. Following studies implicating floral development genes in later fruit maturation, RAP2-7-like genes may also control expression of genes associated with fruit quality.

Many of the observed differentially expressed genes from this study appear to be induced preferentially at different periods of fruit development, suggesting they may be induced in response to unique cues. A larger scale RNA-seq analysis of GD and HC tissues is expected to provide genetic blueprints for unique responses to the stresses encountered by fruit during the production, storage and postharvest chain. While desirable in the retail market, $\mathrm{HC}$ apples exhibit increased frequency of disorders associated with storage conditions relative to other popular cultivars [48]. Identification of these genes may provide an indication of future apple germplasm sensitivity to oxidative stress and frequency of abiotic stress-related storage disorders after harvest. Older varieties such as GD remain popular in the industry due to their unique fruit quality characteristics. In such backgrounds, expression of a senescence-associated gene may mitigate oxidative stress in the fruit at the expense of desirable traits such as acidity, crispiness, and juiciness. Evidence of variability in protein homeostasis activity was also observed among GD and HC tissues from our study. In both sampling time points, GD peel tissues were observed to have higher abundance of SKP1-like and e4-like ubiquitin conjugation factor transcripts (MDP0000213808), than in HC peel and endocarp. As critical components of the 26S-ubiquitin proteolytic pathway, differential abundance of these transcripts may suggest variability in GD and HC post-translational regulation of key points of both ethylene signaling and biosynthesis in fruits [49,50]. We also identified a transcript (MDP0000547450) with homology to UNC93-like genes in higher abundance in GD peel, compared to all HC tissues. Little is known about the role of these proteins in higher plants, or in fruit development. After initial identification in lower plants, UNC93-like genes were found to be involved in regulation of asexual reproduction in Arabidopsis [33]. UNC93-like transcripts were present in seed and silique tissue only, suggesting a role in reproductive tissue development. As with MADS-box genes of the ABC model of floral meristem development, UNC93-like genes may have unknown roles in fruit development [50].

In order to identify the components guiding specific apple fruit traits, differences between cultivars at the genomic, transcriptomic, proteomic, and epigenetic states should be investigated. With respect to probing the changes in apple gene expression, several studies have used microarray analysis of gene expression [1,51,52]. With the issues associated with microarray accuracy and reproducibility [53], expanding genomic resources available for Rosaceae, and increased access to next-generation sequence technologies, RNA-seq is emerging as the gold standard to identify transcriptional differences between apple cultivars. RNA-seq has already been shown to be more sensitive in detecting genetic variants, resolving isoforms, detecting transcripts with low abundance, and had higher sensitivity in detecting differential expression [54]. While the DD method enables identification of differentially expressed genes between apple cultivars, it does have the obvious limitations of being labor intensive and time consuming along with a capacity to capture a limited number of gene fragments. A combined $\mathrm{DD} /$ next-generation sequencing approach may reduce upfront experimental costs and time, while providing useful insights into differential gene expression. 


\section{Conclusions}

Given the widespread adoption of next-generation sequencing approaches, the differential display technique can often be thought of as dated. Here, we show that differential display effectively identified differential expression between samples, and has the potential to complement next-generation sequencing approaches. Out of a total of 105 extracted bands in this experiment, we confirmed differential expression of 31 genes in the peel tissue of "Golden Delicious" and "Honeycrisp" at two important time points in fruit development. Many of these differentially expressed genes encode for potentially important proteins involved in the fruit traits that differ between the two investigated cultivars. Differential expression appears to occur in developmental, cultivar-specific, and potentially seasonally-variable trends. Of these, an intriguing group of transcripts were identified as potential regulators of tissue redox homeostasis and respiration. In general, the roles of many of the identified proteins in both the peroxisome and mitochondria concerning fruit development, ripening regulation, and fruit quality remain unclear. However, these proteins present interesting groups of genes for future characterization.

Supplementary Materials: The following are available online at www.mdpi.com/2311-7524/2/3/11/s1, Table S1: Subset of differentially expressed transcripts from differential display study, confirmed via qRT-PCR, from apple genome release 1.0; Table S2: BLAST output from Blast2GO against the nr-database using sequences of apple genome (v1.0) predicted genes against sequences with highest similarity to sequenced differential display fragments; Table S3: Forward and Reverse primer sequences designed based upon predicted gene sequence from Apple Genome (1.0); Figure S1: Low differential expression of genes identified with differential display; Figure S2: Seasonally-variable differential expression of genes identified with differential display.

Acknowledgments: The authors thank Deb Pehrson for assistance with sample procurement at Washington State University Tukey Orchard, Artemus Harper for assistance with qPCR primer design, Tyson Koepke for assistance in performing differential display, and Salma Tariq, Michela Quickstad, Kiara B. Little, and Emily Rose Rodgers for assistance in cloning differential display fragments. The publication was in part supported by NIH/NIGMS through institutional training grant award T32-GM008336. Its contents are solely the responsibility of the authors and do not necessarily represent the official views of the NIGMS or NIH. USDA-NIFA grant 2008-35300-04676 and ARC Hatch grant WNP00677 to AD are gratefully acknowledged for partial support of the work in this publication.

Author Contributions: S.S. and A.D. designed the study. Samples were obtained by S.S. Samples were prepared by S.S. Sequencing was performed by S.S. and R.F. qRT-PCR was performed by S.S., C.H. and R.F. Data analysis and interpretation was performed by S.S., C.H., R.F. and A.D. The manuscript was prepared by A.D., S.S., C.H. and R.F. All authors reviewed and approved of the final manuscript.

Conflicts of Interest: The authors declare no conflict of interest.

\section{References}

1. Janssen, B.J.; Thodey, K.; Schaffer, R.J.; Alba, R.; Balakrishnan, L.; Bishop, R.; Bowen, J.H.; Crowhurst, R.N.; Gleave, A.P.; Ledger, S.; et al. Global gene expression analysis of apple fruit development from the floral bud to ripe fruit. BMC Plant Biol. 2008. [CrossRef] [PubMed]

2. Kupferman, E. The Role of Ethylene in Determining Apple Harvest and Storage Life. Available online: http:/ / postharvest.tfrec.wsu.edu/pages/N4I1C (accessed on 4 March 2016).

3. Conesa, A.; Gotz, S.; Garcia-Gomez, J.M.; Terol, J.; Talon, M.; Robles, M. Blast2GO: A universal tool for annotation, visualization and analysis in functional genomics research. Bioinformatics 2005, 21, 3674-3676. [CrossRef] [PubMed]

4. Götz, S.; García-Gómez, J.M.; Terol, J.; Williams, T.D.; Nagaraj, S.H.; Nueda, M.J.; Robles, M.; Talón, M.; Dopazo, J.; Conesa, A. High-throughput functional annotation and data mining with the Blast2GO suite. Nucleic Acid Res. 2008, 36, 3420-3435. [CrossRef] [PubMed]

5. Emanuelsson, O.; Brunak, S.; von Heijne, G.; Nielsen, H. Locating proteins in the cell using TargetP, SignalP and related tools. Nat. Protoc. 2007, 2, 953-971. [CrossRef] [PubMed]

6. Nosarzewski, M.; Archbold, D.D. Tissue-specific expression of SORBITOL DEHYDROGENASE in apple fruit during early development. J. Exp. Bot. 2007, 58, 1863-1872. [CrossRef] [PubMed]

7. Zhang, S.; Chen, W.; Xin, L.; Gao, Z.; Hou, Y.; Yu, X.; Zhang, Z.; Qu, S. Genomic variants of genes associated with three horticultural traits in apple revealed by genome re-sequencing. Hortic. Res. 2014, 1, 1-14. [CrossRef] [PubMed] 
8. Zsolt, A.; Deák, C.; Miskó, A.; Tóth, M.; Papp, I. Development of cDNA normalization system and preliminary transcription analysis of KCS genes in apple tissues. Acta Univ. Agric. Silvic. Mendel. Brun. 2014, 59, 9-12.

9. Ruijter, J.M.; Ramakers, C.; Hoogaars, W.M.H.; Karlen, Y.; Bakker, O.; van den Hoff, M.J.B.; Moorman, A.F.M. Amplification efficiency: Linking baseline and bias in the analysis of quantitative PCR data. Nucleic Acid Res. 2009, 37, 1-12. [CrossRef] [PubMed]

10. Pfaffl, M.W. A new mathematical model for relative quantification in real-time RT-PCR. Nucleic Acid Res. 2001, 29, 1-6. [CrossRef]

11. Dixon, J.; Hewett, E.W. Factors affecting apple aroma/flavour volatile concentration: A Review. N. Z. J. Crop Hortic. Sci. 2000, 28, 155-173. [CrossRef]

12. Goulao, L.F.; Santos, J.; de Sousa, I.; Oliveira, C.M. Patterns of enzymatic activity of cell wall-modifying enzymes during growth and ripening of apples. Postharvest Biol. Technol. 2007, 43, 307-318. [CrossRef]

13. Solovchenko, A.E.; Merzlyak, M.N.; Pogosyan, S.I. Light-induced decrease of reflectance provides an insight in the photoprotective mechanisms of ripening apple fruit. Plant Sci. 2010, 178, 281-288. [CrossRef]

14. Emanuelsson, O.; Nielsen, H.; Brunak, S.; von Heijne, G. Predicting subcellular localization of proteins based on their N-terminal amino acid sequence. J. Mol. Biol. 2000, 300, 1005-1016. [CrossRef] [PubMed]

15. Crifò, T.; Puglisi, I.; Petrone, G.; Recupero, G.R.; Piero, A.R.L. Expression analysis in response to low temperature stress in blood oranges: Implication of the flavonoid biosynthetic pathway. Gene 2011, 476, 1-9. [CrossRef] [PubMed]

16. Dietz, K.J.; Vogel, M.O.; Viehhauser, A. AP2/EREBP transcription factors are part of gene regulatory networks and integrate metabolic, hormonal and environmental signals in stress acclimation and retrograde signalling. Protoplasma 2010, 245, 3-14. [CrossRef] [PubMed]

17. Chung, M.Y.; Vrebalov, J.; Alba, R.; Lee, J.; McQuinn, R.; Chung, J.D.; Klein, P.; Giovannoni, J. A tomato (Solanum lycopersicum) APETALA2/ERF gene, SlAP2a, is a negative regulator of fruit ripening. Plant J. 2010, 64, 936-947. [CrossRef] [PubMed]

18. Cai, C.; Xu, C.-J.; Li, X.; Ferguson, I.; Chen, K. Accumulation of lignin in relation to change in activities of lignification enzymes in loquat fruit flesh after harvest. Postharvest Biol. Technol. 2006, 40, 163-169. [CrossRef]

19. Rose, J.K.C.; Bennett, A.B. Cooperative disassembly of the cellulose-xyloglucan network of plant cell walls: Parallels between cell expansion and fruit ripening. Trends Plant Sci. 1999, 4, 176-183. [CrossRef]

20. Louveau, T.; Leitao, C.; Green, S.; Hamiaux, C.; van der Rest, B.; Dechy-Cabaret, O.; Atkinson, R.G.; Chervin, C. Predicting the substrate specificity of a glycosyltransferase implicated in the production of phenolic volatiles in tomato fruit. FEBS J. 2011, 278, 390-400. [CrossRef] [PubMed]

21. Puupponen-Pimiä, R.; Nohnek, L.; Meier, C.; Kähkönen, M.; Heinonen, M.; Hopia, A.; Oksman-Caldentey, K.M. Antimicrobial properties of phenolic compounds from berries. J. Appl. Microbiol. 2001, 90, 494-507. [CrossRef] [PubMed]

22. Flachowsky, H.; Halbwirth, H.; Treutter, D.; Richter, K.; Hanke, M.V.; Szankowski, I.; Gosch, C.; Stich, K.; Fischer, T.C. Silencing of flavanone-3-hydroxylase in apple (Malus $\times$ domestica Borkh.) leads to accumulation of flavanones, but not to reduced fire blight susceptibility. Plant Physiol. Biochem. 2012, 51, 18-25. [CrossRef] [PubMed]

23. Çakir, B.; Agasse, A.; Gaillard, C.; Saumonneau, A.; Delrot, S.; Atanassova, R. A grape ASR protein involved in sugar and abscisic acid signaling. Plant Cell 2003, 15, 2165-2180. [CrossRef] [PubMed]

24. Golan, I.; Guadalupe, P.; Dominguez, Z.K.; Shkolnik-Inbar, D.; Carrari, F.; Bar-Zvi, D. Tomato ABSCISIC ACID STRESS RIPENING (ASR) gene family revisited. PLoS ONE 2014, 9, e107117.

25. Eubel, H.; Meyer, E.H.; Taylor, N.L.; Bussell, J.D.; O’Toole, N.O.; Heazlewood, J.L.; Castleden, I.; Small, I.D.; Smith, S.M.; Millar, A.H. Novel Proteins, putative membrane transporters, and an integrated metabolic network are revealed by quantitative proteomic analysis of Arabidopsis cell culture peroxisomes. Plant Physiol. 2008, 148, 1809-1829. [CrossRef] [PubMed]

26. Kamada, T.; Nito, K.; Hayashi, H.; Mano, S.; Hayashi, M.; Nishimura, M. Functional differentiation of peroxisomes revealed by expression profiles of peroxisomal genes in Arabidopsis thaliana. Plant Cell Physiol. 2003, 44, 1275-1289. [CrossRef] [PubMed]

27. Wiszniewski, A.A.G.; Zhou, W.; Smith, S.M.; Bussell, J.D. Identification of two Arabidopsis genes encoding a peroxisomal oxidoreductase-like protein and an acyl-CoA synthetase-like protein that are required for responses to pro-auxins. Plant Mol. Biol. 2009, 69, 503-515. [CrossRef] [PubMed] 
28. Goda, H.; Sawa, S.; Asami, T.; Fujioka, S.; Shimada, Y.; Yoshida, S. Comprehensive comparison of auxin-regulated and brassinosteroid-regulated genes in Arabidopsis. Plant Physiol. 2004, 134, 1555-1573. [CrossRef] [PubMed]

29. Schweighofer, A.; Hirt, H.; Meskiene, I. Plant PP2C phosphatases: Emerging functions in stress signaling. Trends Plant Sci. 2004, 9, 236-243. [CrossRef] [PubMed]

30. Sharma, B.; Joshi, D.; Yadav, P.K.; Gupta, A.K.; Bhatt, T.K. Role of ubiquitin-mediated degradation system in plant biology. Front. Plant Biol. 2016. [CrossRef] [PubMed]

31. Ascencio-Ibanez, J.T.; Sozzani, R.; Lee, T.J.; Chu, T.M.; Wolfinger, R.D.; Cella, R.; Hanley-Bowdoin, L. Global analysis of Arabidopsis gene expression uncovers a complex array of changes impacting pathogen response and cell cycle during geminivirus infection. Plant Physiol. 2008, 148, 436-454. [CrossRef] [PubMed]

32. Sierla, M.E.; Feys, B.J. Characterization of Arabidopsis thaliana orthologues of GAAP, a Golgi-localized anti-apoptotic protein. In Proceedings of the 20th International Conference on Arabidopsis Research, Edinburgh, UK, 30 June-4 July 2009.

33. Cordle, A.R.; Irish, E.E.; Cheng, C.L. Gene expression associated with apogamy commitment in Ceratopteris richardii. Sex. Plant Reprod. 2012, 25, 293-304. [CrossRef] [PubMed]

34. Foyer, C.H.; Noctor, G. Oxidant and antioxidant signalling in plants: A re-evaluation of the concept of oxidative stress in a physiological context. Plant Cell Environ. 2005, 28, 1056-1071. [CrossRef]

35. Catoni, E.; Desimone, M.; Hilpert, M.; Wipf, D.; Kunze, R.; Schneider, A.; Flügge, U.I.; Schumacher, K.; Frommer, W.B. Expression pattern of a nuclear encoded mitochondrial arginine-ornithine translocator gene from Arabidopsis. BMC Plant Biol. 2003. [CrossRef]

36. Picault, N. Plant mitochondria: From genome to function. In Plant Mitochondrial Carriers; Day, D.A., Millar, A.H., Whelan, J., Eds.; Kluwer Academic Publisher: Dordrecht, The Netherlands, 2004; Volume 17.

37. Solomon, M.; Belenghi, B.; Delledonne, M.; Menachem, E.; Levine, A. The involvement of cysteine proteases and protease inhibitor genes in the regulation of programmed cell death in plants. Plant Cell 1999, 11, 431-443. [CrossRef] [PubMed]

38. Krüger, J.; Thomas, C.M.; Golstein, C.; Dixon, M.S.; Smoker, M.; Tang, S.; Mulder, L.; Jones, J.D.G. A tomato cysteine protease required for Cf-2-dependent disease resistance and suppression of autonecrosis. Science 2002, 296, 744-747. [CrossRef] [PubMed]

39. Costa, F.; Alba, R.; Schouten, H.; Soglio, V.; Gianfranceschi, L.; Serra, S.; Musacchi, S.; Sansavini, S.; Costa, G.; Fei, Z; i et al. Use of homologous and heterologous gene expression profiling tools to characterize transcription dynamics during apple fruit maturation and ripening. BMC Plant Biol. 2010, 10, 1-17. [CrossRef] [PubMed]

40. Gosch, G.; Halbwirth, H.; Schneider, B.; Hölscher, D.; Stich, K. Cloning and heterologous expression of glycosyltransferases from Malus $\times$ domestica and Pyrus communis, which convert phloretin to phloretin 2'-O-glucoside (phloridzin). Plant Sci. 2010, 178, 299-306. [CrossRef]

41. Blanco-Portales, R.; Medina-Escobar, N.; López-Ráez, J.A.; González-Reyes, J.A.; Villalba, J.M.; Moyano, E.; Caballero, J.L.; Muñoz-Blanco, J. Cloning, expression and immunolocalization pattern of a cinnamyl alcohol dehydrogenase gene from strawberry (Fragaria $\times$ ananassa cv. Chandler). J. Exp. Bot. 2002, 53, 1723-1734. [CrossRef] [PubMed]

42. Salentijn, E.M.J.; Aharoni, A.; Schaart, J.G.; Boone, M.J.; Krens, F.A. Differential gene expression analysis of strawberry cultivars that differ in fruit firmness. Physiol. Plant. 2003, 118, 571-578. [CrossRef]

43. Redgwell, R.J.; Fry, S.C. Xyloglucan endotransglycosylase activity increases during kiwifruit (Actinidia deliciosa) ripening (Implications for fruit softening). Plant Physiol. 1993, 103, 1399-1406. [PubMed]

44. Cutillas-Iturralde, A.; Zarra, I.; Fry, S.C.; Lorences, E.P. Implication of persimmon fruit hemicellulose metabolism in the softening process. Importance of xyloglucan endotransglycosylase. Physiol. Plant. 2006, 91, 169-176. [CrossRef]

45. Gutiérrez-Larraínzara, M.; Rúaa, J.; Carob, I.; de Castroa, C.; de Arriagaa, D.; García-Armestob, M.R.; del Valle, P. Evaluation of antimicrobial and antioxidant activities of natural phenolic compounds against foodborne pathogens and spoilage bacteria. Food Control 2012, 26, 555-563. [CrossRef]

46. Osorio, S.; Scossa, F.; Fernie, A.R. Molecular regulation of fruit ripening. Front. Plant Sci. 2013, 198, 1-8. [CrossRef] [PubMed]

47. Pattison, R.J.; Csukasi, F.; Catalá, C. Mechanisms regulating auxin action during fruit development. Physiol. Plant. 2014, 151, 62-72. [CrossRef] [PubMed] 
48. Watkins, C.B.; Nock, J.F.; Weis, S.A.; Jayanty, S.; Beaudry, R.M. Storage temperature, diphenylamine, and pre-storage delay effects on soft scald, soggy breakdown and bitter pit of "Honeycrisp" apples. Postharvest Biol. Technol. 2004, 32, 213-221. [CrossRef]

49. Cherian, S.; Figueroa, C.R.; Nair, H. "Movers and Shakers" in the regulation of fruit ripening: A cross-dissection of climacteric versus non-climacteric fruit. J. Exp. Bot. 2014, 65, 4705-4722. [CrossRef] [PubMed]

50. Ireland, H.S.; Yao, J.-L.; Tomes, S.; Sutherland, P.W.; Nieuwenhuizen, N.; Gunaseelan, K.; Winz, R.A.; David, K.M.; Schaffer, R.J. Apple SEPALLATA1/2-like genes control fruit flesh development and ripening. Plant J. 2013, 73, 1044-1056. [CrossRef] [PubMed]

51. Soria-Guerra, R.E.; Rosales-Mendoza, S.; Gasic, K.; Wisniewski, M.E.; Band, M.; Korban, S.S. Gene expression is highly regulated in early developing fruit of apple. Plant Mol. Biol. Rep. 2011, 29, 885-897. [CrossRef]

52. Zhu, Y.; Zheng, P.; Varanasi, V.; Shin, S.; Main, D.; Curry, E.; Mattheis, J.P. Multiple plant hormones and cell wall metabolism regulate apple fruit maturation patterns and texture attributes. Tree Genet. Genomes 2012, 8 , 1389-1406. [CrossRef]

53. Draghici, S.; Khatri, P.; Eklund, A.C.; Szallasi, Z. Reliability and reproducibility issues in DNA microarray measurements. Trends Genet. 2006, 22, 101-109. [CrossRef] [PubMed]

54. Zhao, S.; Fung-Leung, W.-P.; Bittner, A.; Ngo, K.; Liu, X. Comparison of RNA-seq and microarray in transcriptome profiling of activated T cells. PLOS ONE 2014, 9, e78644. [CrossRef] [PubMed]

(C) 2016 by the authors; licensee MDPI, Basel, Switzerland. This article is an open access article distributed under the terms and conditions of the Creative Commons Attribution (CC-BY) license (http://creativecommons.org/licenses/by/4.0/). 